\title{
Tube Width Fluctuations in F-Actin Solutions
}

\author{
J. Glaser, ${ }^{*}$ D. Chakraborty, and K. Kroy \\ Institut für Theoretische Physik, Universität Leipzig, PF 100920, 04009 Leipzig, Germany
}

\author{
I. Lauter, M. Degawa, ${ }^{\dagger}$ N. Kirchgeßner, B. Hoffmann, R. Merkel, and M. Giesen \\ Institut für Bio- und Nanosysteme: Biomechanik (IBN4), Forschungszentrum Jülich, 52425 Jülich, Germany \\ (Received 15 October 2009; published 13 July 2010)
}

\begin{abstract}
We determine the statistics of the local tube width in F-actin solutions, beyond the usually reported mean value. Our experimental observations are explained by a segment fluid theory based on the binary collision approximation. In this systematic generalization of the standard mean-field approach, effective polymer segments interact via a potential representing the topological constraints. The analytically predicted universal tube width distribution with a stretched tail is in good agreement with the data.
\end{abstract}

PACS numbers: 61.25.H-, 82.35.Pq, 87.16.Ln

The Edwards tube model provides a simple phenomenological description of the complicated topological constraints in entangled solutions of flexible polymers [1]. Using scaling arguments, Odijk [2], Semenov [3], and others have generalized the idea to stiff polymers with a persistence length $l_{p}$ larger than the characteristic backbone length between mutual collisions, which plays the role of the entanglement length in this context. Stiff polymers constitute an important and biologically relevant class of polymers, as they represent the major structural and stress-bearing units of the cytoskeleton of animal cells in the form of filamentous actin and microtubules [4]. Their large $l_{p}$ and contour length $L$ (on the order of $10 \mu \mathrm{m}$ for actin) have opened the possibility of direct microscopic visualizations of the tube [5]. Beyond its intuitive appeal and obvious relevance to single filament experiments in entangled solutions [6,7], the tube enters explicit calculations of the overall mechanical properties of stiff polymer solutions [8-10]. The latter have been measured rheometrically for biopolymers such as actin [11-14] and pectin [15], as well as for self-assembling synthetic networks $[16,17]$. While theoretical approaches so far have employed a homogeneity (or mean-field) assumption, treating the tube radius as a constant, experiments [5$7,18,19]$ indicate substantial heterogeneities, which recently also have been found in simulations [9].

In this Letter, we present a systematic study of these heterogeneities in F-actin solutions, which we quantify in terms of the local tube radius profile $R(s)$ [cf. Figs. 1(a)-1(c)]. From $R(s)$ we infer the entanglement length (Fig. 4) and, independently, the tube radius distribution $P(R)$ [Fig. 1(d)]. The latter plays a similar role as the distribution of void spaces or pores in other disordered materials, such as packings of grains or colloidal particles [20]. As a main result, $P(R)$ is found to be described by a universal master function with a stretched Gaussian tail (Fig. 3, inset). We develop a systematic theory of tube fluctuations that explains our data and provides the basis for a more comprehensive characterization of the structural and elastic properties of stiff polymer solutions than possible within the conventional mean-field theory [8].

F-actin solutions were prepared at various monomer concentrations $c$ [21]. Rhodamine-phalloidin labeled solutions were mixed with unlabeled solutions at a ratio of 1:1000. Time series of typically 150 pictures of individual labeled filaments were recorded using an inverse confocal microscope (LSM510, Carl Zeiss Jena, Germany). These
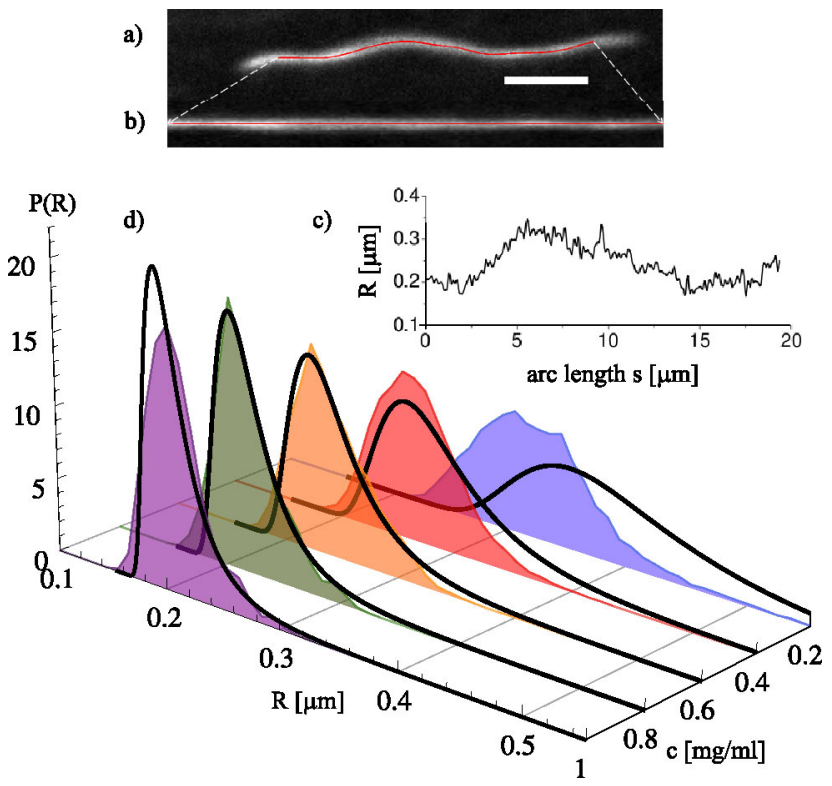

FIG. 1 (color online). (a) Superimposed confocal microscopy images of a fluorescent actin filament in a background solution and a spline representing the tube backbone; scale bar, $5 \mu \mathrm{m}$; (b) rectified image; (c) tube radius profile $R(s)$ determined as standard deviation from Gaussian fits to the transverse intensity profile; (d) normalized tube radius distribution $P(R)$ obtained from cumulative contour lengths of 536, 804, 301, 225, and $116 \mu \mathrm{m}$ for actin concentrations $c=0.2,0.4,0.6,0.8$, $1.0 \mathrm{mg} / \mathrm{ml}$, respectively. Solid lines represent a global fit by Eq. (3) with $\rho=5.95 \mu \mathrm{m}^{-2} c[\mathrm{mg} / \mathrm{ml}]$ and $L=0.91 L_{e}^{\infty}$, including corrections accounting for the line spread function [21]. 
pictures were superimposed to obtain a time-averaged image of the fluctuating test filament, where intensity reflects the residence time of the filament [Fig. 1(a)]. Smooth tube contours connecting points of maximum intensity were constructed subject to a curvatureminimization constraint. The local tube radius $R(s)$ was identified with the standard deviation of Gaussians fitted to the transverse intensity profiles of a rectified tube image, as exemplified in Figs. 1(b) and 1(c) [21]. Along a single test filament, $R(s)$ exhibits pronounced fluctuations, which were binned to sample the tube radius distribution $P(R)$. The result is depicted in Fig. 1(d) (shaded areas) for various actin concentrations $c$. The peak position and width, corresponding to the typical value and the fluctuations of $R$, respectively, are seen to decrease with increasing $c$. Yet, as we demonstrate below, the non-Gaussian skewed and leptokurtic shape of $P(R)$ is well described by a $c$-independent master function (Fig. 3, inset).

We develop our theoretical approach along the lines of the binary collision approximation (BCA) [8,9]. It replaces the complicated topological constraints in an entangled polymer solution by an effective model: an individual test polymer of length $L$ in a tubelike harmonic confinement potential $\bar{\phi} \mathbf{h}^{2} / 2$ per unit length, where $\mathbf{h}(s)$ parametrizes the transverse contour undulations along the backbone. In a self-consistent pair approximation, the tube potential is required to represent the cumulative effect of independent pair collisions with the background polymers. These collisions are not to be understood as bare microscopic encounters of polymer backbones, though, but rather refer to effective tube collisions. The bare interactions caused by the impenetrability of the polymer backbones are coarse-grained over the fast local conformational fluctuations of two colliding polymers confined to their tubes, which prescribe a "quenched" geometry for them. The corresponding averages in a given tube configuration and over different tube configurations are represented by brackets $\langle\cdots\rangle_{\bar{\phi}}$ and over-bars $\cdots$, respectively. The overbar on the tube stiffness $\bar{\phi}$ thus indicates that the latter represents the cumulative contribution from collisions in all possible geometries and topologies as opposed to collisions in a prescribed tube configuration. A subtlety in counting states is that the topology of stiff but bendable polymers, as opposed to rigid rods, is not uniquely determined by the center-of-mass positions and orientations. Conversely, in any given topology $\sigma= \pm$ (or "above" and "below"), positive and negative "distances" $x \lessgtr 0$ need to be distinguished, as sketched for $\sigma=+$ in Fig. 2(a).

The wormlike chain model in the weakly bending rod limit with eigenmodes $\mathbf{h}(q)$ of the undulations $\mathbf{h}(s)$ is employed. Equipartition yields $\left\langle\mathbf{h}(q) \cdot \mathbf{h}\left(q^{\prime}\right)\right\rangle_{\bar{\phi}}=2 \delta(q+$ $\left.q^{\prime}\right) /\left(l_{p} q^{4}+\bar{\phi}\right)$ for $L \gg L_{e}^{\infty}$, where $L_{e}^{\infty} \equiv\left(l_{p} / \bar{\phi}\right)^{1 / 4}$ is the mean-field entanglement length [8]. Natural units with $k_{B} T=1$, so that $l_{p}$ is synonymous with the bending rigidity, are used throughout. The mean-field (projected) tube

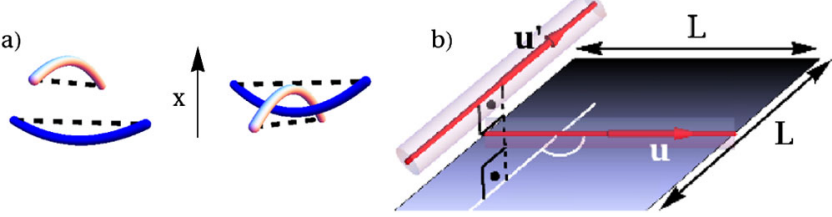

FIG. 2 (color online). (a) A pair of colliding filaments is assigned a "distance" $x$ and "topology" $\sigma= \pm$ as exemplified for $\sigma=+$ with $x>0$ (left) and $x<0$ (right). A reflection at the horizontal midplane amounts to $x \rightarrow-x, \sigma \rightarrow-\sigma$. (b) Overlap region (shaded) for two segments of length $L$ with orientations $\mathbf{u}, \mathbf{u}^{\prime}$ enclosing an arbitrary angle.

radius then follows as $R^{\infty} \equiv R(\bar{\phi})$, given by the function

$$
R^{2}(\phi) \equiv \int \frac{d s}{2 L}\left\langle\mathbf{h}^{2}\right\rangle_{\phi}=2^{-3 / 2} l_{p}^{-1 / 4} \phi^{-3 / 4}
$$

evaluated at $\phi=\bar{\phi}$. Similarly, one gets the partition sum for the Gaussian contour undulations $\mathbf{h}(s)$ [8]. Its negative logarithm is the mentioned coarse-grained interaction or "BCA potential" $\quad F_{\sigma}(x)=-\ln \left\{\operatorname{erfc}\left[-2^{-1 / 2} \sigma x /\left(R_{0}^{2}+\right.\right.\right.$ $\left.\left.\left.R_{1}^{2}\right)^{1 / 2}\right] / 2\right\}$ between two tubes of radii $R_{0}$ and $R_{1}$ at separation $x$ along the direction of nearest approach. If we allow for a uniform transverse displacement $h$ of the test tube at an angle $\psi$ relative to the $x$ direction, which does not change the topology, the contribution to the confinement strength resulting from collisions in the prescribed tube configuration follows as $\phi=\left.L^{-1} \partial_{h}^{2} F_{\sigma}(x-h \cos \psi)\right|_{h=0}$. In mean-field approximation, setting $R_{0}=R_{1} \equiv R^{\infty}$ and performing the configurational average [8] yields the tube strength $\bar{\phi}$ as a function of $R^{\infty}$ and the line concentration $\rho$ (polymer length per volume). From Eq. (1), $R^{\infty}=$ $0.66 \rho^{-3 / 5} l_{p}^{-1 / 5}$ is finally self-consistently determined [21].

The conventional BCA, as a mean-field theory, is exclusively concerned with the average values $\bar{\phi}$ and $R^{\infty}$. To get hold of the measured spatial tube width fluctuations, we introduce a canonical ensemble of $N+1$ independent entanglement segments of length $L$ characterized by their individual fields $\phi_{i}$ and corresponding tube radii $R_{i}$ before averaging over the segment ensemble. In a formal generalization of the BCA that we call the segment fluid model, any overlapping pair in the ensemble interacts with the BCA pair potential, now written as $V_{\sigma}(x) \equiv$ $\chi\left(\mathbf{r}, \mathbf{u}, \mathbf{u}^{\prime}\right) F_{\sigma}(x)$ with the characteristic function $\chi$ of the overlap between two segments with orientations $\mathbf{u}, \mathbf{u}^{\prime}$ separated by r. As depicted in Fig. 2(b), the two segments are said to "collide" or to "overlap" if the projection of the center-of-mass of the segment with orientation $\mathbf{u}^{\prime}$ onto the $\mathbf{u}-\mathbf{u}^{\prime}$ plane falls into the shaded area with edges of length $L$ and the segment with orientation $\mathbf{u}$ at its center. All pairs of segments are assigned a binary topological state variable $\sigma_{i j}= \pm$. The confinement potential for a test segment with index 0 is then computed as the cumulative effect from the collisions with all overlapping segments. Explicitly, after averaging over the uniformly distributed angles $\psi_{0 j}$ of perturbing displacements of the test segment, 
its individual tube strength is written as a local field $\phi_{0}=\sum_{i=1}^{N} \phi_{0 i}$ with $\phi_{0 i}=(2 L)^{-1} V_{\sigma_{0 i}}^{\prime \prime}\left(x_{0 i}\right)$. The average tube strength $\bar{\phi}$ is then obtained by taking the configurational average, i.e., by summing $\phi_{0}$, weighted by the Boltzmann factor for all pair interactions, over all topologies $\sigma_{i j}$ and positions and orientations of the segments.

We now apply this formalism to calculate the distribution $P(\phi)$ of tube strengths. For the average over topologies we exploit the identity $e^{-V_{+}}+e^{-V_{-}}=1$ for mutually overlapping segments, such that Boltzmann factors not involving the test segment cancel out in the partition sum. This is a consequence of the topological origin of the effective pair interactions. Moreover, due to the pair approximation, all interacting pairs are equivalent so that only one representative collision needs to be considered explicitly. In the thermodynamic limit $N \rightarrow \infty$, the characteristic function $\tilde{P}(t)=\overline{e^{i t \phi}}$ of $P(\phi)$ then follows from the identity $(1+x / N)^{N} \rightarrow e^{x}$ as

$$
\tilde{P}(t)=\exp \left[n \int d \mathbf{r}_{1} \frac{d \mathbf{u}_{1}}{4 \pi}\left(\sum_{\sigma} e^{i t \phi_{01}-V_{\sigma}\left(x_{01}\right)}-1\right) \chi\right]
$$

with the segment concentration $n \equiv(N+1) / V=\rho / L$. By a numerical inverse Fourier transform, $P(\phi)$ is obtained, which we recognize as the Holtzmark local field distribution of uncorrelated particles [22]. The tube radius distribution $P(R)$ then follows from $P(\phi)$ by applying Eq. (1). In Fig. 3 (solid lines) it is shown for the special case of a pair potential $V_{\sigma}$ with a common average range $R_{i} \equiv \bar{R}$ for all segments $i$.

To validate the result, numerical (Monte Carlo) integration of a fluid of effective segments under the same assumptions as used in the theory was performed [21] [see Fig. 3 (dots)]. An examination of the numerical results establishes that the underlying asymmetric distribution $P(\phi)$ is well approximated by a gamma distribution $\Gamma_{k, \theta}(\phi)$. Its shape and scale parameters, $k=4.013 \rho L \bar{R}$ and $\theta=0.125\left(L \bar{R}^{2}\right)^{-1}$, are determined by matching its first two cumulants with the predictions from Eq. (2). Via Eq. (1), an analytical expression for $P(R)$ ensues,

$$
P(R)=\frac{8}{3 R(k-1) !} \exp (-y) y^{k}, \quad y \equiv \frac{1}{4 l_{p}^{1 / 3} R^{8 / 3} \theta} .
$$

It is compared with our numerical results for a fixed prescribed segment length $L$ and various reduced concentrations $\rho L \bar{R}$ in Fig. 3.

What remains to be done is to identify the physical meaning of the effective segment length $L$. Qualitatively, one expects it to be equal to the mean-field entanglement length $L_{e}^{\infty}=\left(l_{p} / \bar{\phi}\right)^{1 / 4}$. The latter may in turn be expressed in terms of the mean-field tube radius $R^{\infty}$ and the line concentration $\rho$, respectively. Via Eq. (1), $L_{e}^{\infty}=$ $\sqrt{2}\left(R^{\infty}\right)^{2 / 3} l_{p}^{1 / 3} \propto \rho^{-2 / 5}$. For the natural choice of $L=$ $L_{e}^{\infty}$, the mean tube radius $\bar{R} \equiv \overline{R(\phi)}$ follows from a numerical solution of the implicit equation $\bar{R}=\int d R R P(R)$ as $\bar{R}=1.15 R^{\infty}$. Its close match with the mean-field value

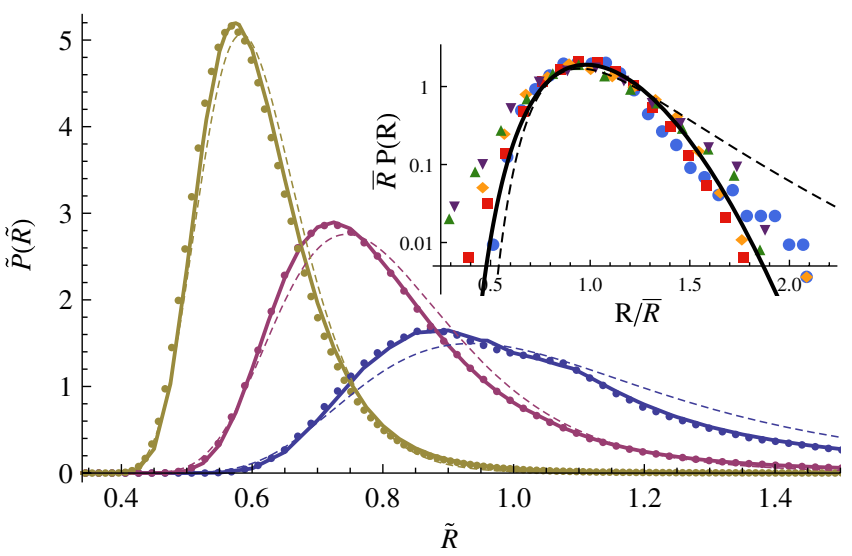

FIG. 3 (color online). Dimensionless tube radius distribution $\tilde{P}(\tilde{R})(\tilde{P} d \tilde{R}=P d R)$ with scaling variable $\tilde{R} \equiv L^{-3 / 8} \bar{R}^{-3 / 4} l_{p}^{1 / 8} R$ for reduced concentrations $\rho L \bar{R}=0.5,1,2$ (bottom to top): Holtzmark distribution, Eq. (2) (solid line); gamma distribution approximation, Eq. (3) (dashed line); numerical integration (dotted line). A bimodal structure develops if the theory is pushed towards the (unphysical) limit $\rho L \bar{R} \rightarrow 0$. Inset: Rescaled distribution as a function of $R / \bar{R}$ in semilogarithmic representation: experimental data for $c=0.2$ (circles), 0.4 (squares), 0.6 (diamonds), 0.8 (upright triangles), and $1.0 \mathrm{mg} / \mathrm{ml}$ (downward-facing triangles), analytical approximation from Eq. (3) as in Fig. 1 (dashed line), and self-consistent theory described in the main text with a slightly renormalized value of the segment length $L=1.62 L_{e}$ (solid line).

$R^{\infty}=\lim _{L \rightarrow \infty} \bar{R}$ suggests to parametrize the distribution $P(R)$ by the mean-field value $R^{\infty} \propto \rho^{-3 / 5}$ for $\bar{R}$, with negligible error. The shape parameter $k$ thereupon becomes independent of $\rho$ and only depends on $L / L_{e}^{\infty}$. The corresponding predictions of Eq. (3) compare favorably with the measured tube radius distribution, as demonstrated in Fig. 1(d). In this comparison, $L / L_{e}^{\infty}$ and $\rho / c$ are used as global concentration-independent fit parameters. While the fit does indeed corroborate the expectation $L / L_{e}^{\infty} \approx 1, \rho / c$ turns out to be about a factor of 6.6 smaller than estimated from the molecular weight and structure of monomeric actin [8]. This numerical discrepancy can be eliminated without significant consequences for the quality of the fit and the value of $L / L_{e}^{\infty}$ [23].

Note that Eq. (3) implies that the concentration dependence enters the tube radius distribution only through the average tube radius $\bar{R}$, such that $\bar{R} P(R)$ defines a concentration-independent master function of $R / \bar{R}$. The inset of Fig. 3 (symbols) demonstrates that the data indeed scale satisfactorily. The semilogarithmic representation reveals some systematic deviation of Eq. (3) (dashed) from the data, however. This shortcoming is due to the preaveraging $R_{i} \equiv \bar{R}$ employed in the derivation of Eq. (3), and can be overcome by evaluating our systematic theory more accurately. To this end, the variable tube radius $R_{0}$ of the test segment in the BCA potential is self-consistently identified with the argument $R$ of the distribution $P(R)$, and only the radius $R_{1}$ of the representative collision partner is preaveraged. This amounts to replacing $\bar{R}$ in 

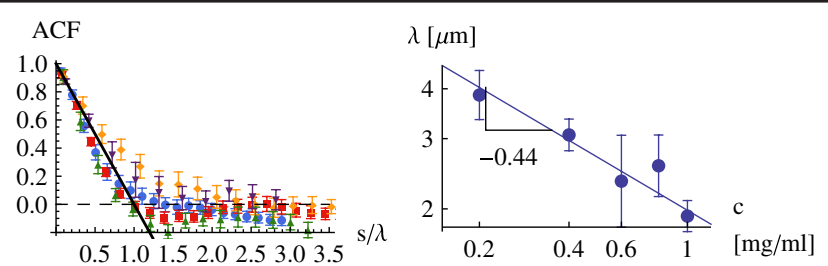

FIG. 4 (color online). Left: Autocovariance function (ACF) of the tube radius profile averaged over 24, 37, 18, 13, and 9 filaments for $c=0.2,0.4,0.6,0.8$, and $1.0 \mathrm{mg} / \mathrm{ml}$, respectively (symbols as in Fig. 3, inset), with the abscissas rescaled by their initial slope $\lambda^{-1}$ (obtained by quadratic extrapolation for a varying fit interval $s=s_{0}, \ldots, s_{\max }, s_{\max } \rightarrow 0$ [21]). Because of the finite optical resolution of the microscope, data points below $s_{0}=230 \mathrm{~nm}$ were not considered. Right: $\lambda$ versus actin concentration $c$ with best power-law fit (exponent $-0.44 \pm$ $0.09)$.

Eq. (3) by $2^{-1 / 2}\left[R^{2}+\left(R^{\infty}\right)^{2}\right]^{1 / 2}$, before substituting $\rho=$ $\left(R^{\infty} / 0.66\right)^{-5 / 3} l_{p}^{-1 / 3}$. Moreover, the segment length $L$ is taken to be proportional to the local entanglement length, $L_{e}=\sqrt{2} R^{2 / 3} l_{p}^{1 / 3}$. The resulting normalized tube radius distribution (solid line in the inset of Fig. 3) is in excellent agreement with our experimental data. It exhibits (up to a spurious logarithm) a tail $P(R) \propto \exp \left[-(R / \bar{R})^{5 / 3}\right]$, similar to what has recently been proposed on empirical grounds [19].

A further consistency check for the developed theory is provided by the spatial autocorrelation of the tube radius profile in Fig. 1(c). It should decay over a characteristic length scale comparable to the entanglement length. The rescaled autocovariance functions determined from a large number of tube radius profiles for various concentrations are shown in Fig. 4 (left panel), rescaled with their initial slope $\lambda^{-1}$. Indeed, the concentration scaling of $\lambda$ thus obtained (right panel) compares favorably with the expectation $\lambda \simeq L_{e}^{\infty} \propto c^{-2 / 5}$.

The fact that heterogeneities give rise to stretched tails in $P(R)$ underscores the importance of shifting the attention from characteristic "numbers" for $R, L_{e}$, etc. [11], to their skewed leptokurtic distributions. As demonstrated above, these are readily accessible in our BCA-based segment fluid model. For instance, subtle nonsteric corrections to the value of the tube radius $R$, e.g., due to transient electrostatic attraction mediated by divalent counterions [24], could systematically be studied by means of $P(R)$ in the future. Our combined experimental and theoretical results might also hold the key to a microscopic explanation of the ubiquitously observed broad distribution of microrheological plateau moduli $[18,25]$.

This work was supported by the Deutsche Forschungsgemeinschaft (DFG) through FOR 877 and the Leipzig School of Natural Sciences-Building with Molecules and Nano-objects. M. D. acknowledges financial support by the Alexander von Humboldt foundation. *jens.glaser@itp.uni-leipzig.de

${ }^{\dagger}$ Present address: Laboratory of Molecular Biophysics, Brain Science Institute, RIKEN-Wako, Saitama 2-1, Japan.

[1] S. F. Edwards, Proc. Phys. Soc. London 92, 9 (1967).

[2] T. Odijk, Macromolecules 16, 1340 (1983).

[3] A. N. Semenov, J. Chem. Soc., Faraday Trans. 2 82, 317 (1986).

[4] K. Kasza, A. Rowat, J. Liu, T. Angelini, C. Brangwynne, G. Koenderink, and D. Weitz, Curr. Opin. Cell Biol. 19, 101 (2007).

[5] J. Käs, H. Strey, and E. Sackmann, Nature (London) 368 , 226 (1994).

[6] M. A. Dichtl and E. Sackmann, New J. Phys. 1, 18 (1999).

[7] M. Romanowska, H. Hinsch, N. Kirchgessner, M. Giesen, M. Degawa, B. Hoffmann, E. Frey, and R. Merkel, Europhys. Lett. 86, 26003 (2009).

[8] D. C. Morse, Phys. Rev. E 63, 031502 (2001).

[9] H. Hinsch, J. Wilhelm, and E. Frey, Eur. Phys. J. E 24, 35 (2007).

[10] P. Fernández, S. Grosser, and K. Kroy, Soft Matter 5, 2047 (2009).

[11] M. Tassieri, R. M. L. Evans, L. Barbu-Tudoran, G. Nasir Khaname, J. Trinick, and T. A. Waigh, Phys. Rev. Lett. 101, 198301 (2008).

[12] B. Hinner, M. Tempel, E. Sackmann, K. Kroy, and E. Frey, Phys. Rev. Lett. 81, 2614 (1998).

[13] M. L. Gardel, M. T. Valentine, J. C. Crocker, A. R. Bausch, and D. A. Weitz, Phys. Rev. Lett. 91, 158302 (2003).

[14] J. Liu, M. L. Gardel, K. Kroy, E. Frey, B. D. Hoffman, J. C. Crocker, A. R. Bausch, and D. A. Weitz, Phys. Rev. Lett. 96, 118104 (2006).

[15] R. R. Vincent, D. N. Pinder, Y. Hemar, and M. A. K. Williams, Phys. Rev. E 76, 031909 (2007).

[16] G. M.H. Wilkins, P.T. Spicer, and M. J. Solomon, Langmuir 25, 8951 (2009).

[17] M. Kühne and C. Friedrich, Rheol. Acta 48, 1 (2009).

[18] Y. Luan, O. Lieleg, B. Wagner, and A. Bausch, Biophys. J. 94, 688 (2008).

[19] B. Wang, J. Guan, S. M. Anthony, S. C. Bae, K. S. Schweizer, and S. Granick, Phys. Rev. Lett. 104, 118301 (2010).

[20] S. Torquato, Random Heterogeneous Materials (Springer, New York, 2002).

[21] See supplementary material at http://link.aps.org/ supplemental/10.1103/PhysRevLett.105.037801 for information about experimental procedures, numerics, and theory details.

[22] S. H. Simon, V. Dobrosavijecić, and R. M. Stratt, J. Chem. Phys. 93, 2640 (1990).

[23] As we will demonstrate elsewhere, the theory can be systematically extended to account for contributions of collective modes and partial shear-induced nematic ordering of the filaments in the experiment, which lead to large $R$ (small $\rho / c$ ) values and explain the mismatch between data and theory for small $c$ in Fig. 1(d). (unpublished results).

[24] J. He, J. Viamontes, and J. X. Tang, Phys. Rev. Lett. 99, 068103 (2007).

[25] T. A. Waigh, Rep. Prog. Phys. 68, 685 (2005). 